\title{
Methamphetamine Self-Administration and Voluntary Exercise Have Opposing Effects on Medial Prefrontal Cortex Gliogenesis
}

\author{
Chitra D. Mandyam, ${ }^{1}$ Sunmee Wee, ${ }^{1}$ Amelia J. Eisch, ${ }^{2}$ Heather N. Richardson, ${ }^{1}$ and George F. Koob ${ }^{1}$ \\ ${ }^{1}$ Committee on the Neurobiology of Addictive Disorders, The Scripps Research Institute, La Jolla, California 92037, and ${ }^{2}$ Department of Psychiatry, \\ University of Texas Southwestern Medical Center, Dallas, Texas 75390
}

Psychostimulant abuse produces deficits in prefrontal cortex (PFC) function, whereas physical activity improves PFC-dependent cognition and memory. The present study explored the vulnerability of medial PFC (mPFC) precursor proliferation and survival to methamphetamine self-administration and voluntary exercise, factors that may have opposing effects on mPFC plasticity to facilitate functional consequences. Intermittent $1 \mathrm{~h}$ access to methamphetamine (I-ShA) increased, but daily 1 and $6 \mathrm{~h}$ access decreased, proliferation and survival, with dose-dependent effects on mature cell phenotypes. All groups showed increased cell death. Voluntary exercise enhanced proliferation and survival but, in contrast to methamphetamine exposure, did not alter cell death or mature phenotypes. Furthermore, enhanced cell survival by I-ShA and voluntary exercise had profound effects on gliogenesis with differential regulation of oligodendrocytes versus astrocytes. In addition, new cells in the adult mPFC stain for the neuronal marker neuronal nuclear protein, although enhanced cell survival by I-ShA and voluntary exercise did not result in increased neurogenesis. Our findings demonstrate that mPFC gliogenesis is vulnerable to psychostimulant abuse and physical activity with distinct underlying mechanisms. The susceptibility of mPFC gliogenesis to even modest doses of methamphetamine could account for the pronounced pathology linked to psychostimulant abuse.

Key words: medial prefrontal cortex; methamphetamine; self-administration; Ki-67; bromodeoxyuridine; voluntary exercise

\section{Introduction}

The ability of the brain to endlessly generate new precursor or neural stem cells throughout adulthood that maintain the structure and function of the adult mammalian brain changed the dogma that new neurons were born only during development (Gould et al., 1999a; Markakis and Gage, 1999; Kempermann, 2002; van Praag et al., 2002; Ramirez-Amaya et al., 2006). Medial prefrontal cortex (mPFC) precursors are mostly gliogenic, and their neurogenic capacity is uncertain and unconfirmed (Gould et al., 1999b; Magavi et al., 2000; Nowakowski and Hayes, 2000; Kornack and Rakic, 2001; Bernier et al., 2002; Ehninger and Kempermann, 2003; Koketsu et al., 2003; Kodama et al., 2004; Dayer et al., 2005; Madsen et al., 2005; Czeh et al., 2007; Ongur et al.,

\footnotetext{
Received June 3, 2007; revised Sept. 7, 2007; accepted Sept. 7, 2007.

This work was supported by National Institute on Drug Abuse Grants DA010072 (G.F.K.) and DA016765 (A.J.E.) and National Institute of Child Health and Human Development Grant HD36460 (Michael R. Gorman). Dr. Michael R. Gorman (University of California, San Diego, La Jolla, CA) kindly provided the Nalgene activity wheels. We acknowledge the excellent technical assistance of Mike Pham, Hanan Jammal, and Krisha Begalla from the independent study program at University of California, San Diego and Simon Huynh and Guadalupe Coronado from the Harvey Mudd College Upward Bound Program (Claremont, CA). We thank Elena Crawford and Drs. Xiaoying Lu and Donna L. Gruol for assistance with microscopy. We appreciate the technical support of Robert Lintz, Yanabel Grant, and Dr. Thomas Greenwell; the editorial assistance of Mike Arends; and critical comments from Dr. Olivier George. This is publication number 18790 from The Scripps Research Institute.

Correspondence should be addressed to Dr. Chitra D. Mandyam, Committee on the Neurobiology of Addictive

Disorders, SP30-2400, The Scripps Research Institute, La Jolla, CA 92037. E-mail: cmandyam@scripps.edu. D0I:10.1523/JNEUROSCI.2505-07.2007

Copyright $\odot 2007$ Society for Neuroscience $\quad 0270-6474 / 07 / 2711442-09 \$ 15.00 / 0$
}

2007). However, evidence suggests that glia (astrocytes and oligodendrocytes) are necessary to maintain the structure and proper functioning of neurons and may in certain enriched environments support neurogenesis of neural stem cells (AlvarezBuylla et al., 2000; Kondo and Raff, 2000; Alvarez-Buylla and Lim, 2004; Sanai et al., 2004; Benarroch, 2005). The involvement of the PFC in both the development and consequences of addiction (Jentsch et al., 2000; Rhodes et al., 2005) raises the hypothesis of a potential role of the newly generated glia in such functional changes.

Drugs of abuse such as psychostimulants are reinforcing with high abuse potential, and methamphetamine is robust in this respect. The PFC has been implicated in several neurobiological phenomena associated with compulsive drug seeking (Rhodes et al., 2005). Despite the potential role of the frontal cortex in compulsive drug seeking (Jentsch et al., 2000), very little information exists about drug intake in the context of compulsive drug use altering precursor cell birth and death in the mPFC. Compulsive intake or escalation of intravenous drug self-administration is observed in rodents with extended access to psychostimulants (Ahmed and Koob, 1998; Kitamura et al., 2006). Therefore, intravenous self-administration holds significant clinical relevance and may provide a useful approach to understanding the neurobiological mechanisms responsible for the transition from drug use to escalation in intake (Ahmed and Koob, 1998). Importantly, the voluntary behavioral paradigm for compulsive drug 
intake may represent a particularly suitable model for testing the hypothesis that alteration in adult $\mathrm{mPFC}$ plasticity by the drug is partly responsible for the addictive behavior. The recent conceptualization of a critical role for deficits in the PFC in promoting addiction-related deficits in executive function (Goldstein and Volkow, 2002) suggests that understanding the role of gliogenesis in various stages of drug taking will likely enhance our ability to understand the pathophysiology of addiction.

Unlike psychostimulants that negatively impact the neurochemistry and neurocircuitry of the mPFC, exercise performed voluntarily has profound positive effects on frontal cortexdependent behavior (Pinilla, 2006; Small et al., 2006; Yu et al., 2006). Such outcomes may be attributable to explicit alterations in PFC cytoarchitecture and plasticity. Therefore, in this study, we incorporated voluntary models of methamphetamine administration and exercise to compare and contrast the vulnerability of the mPFC proliferative environment to negative and positive environmental factors.

\section{Materials and Methods}

Animals, bromodeoxyuridine injections, and tissue preparation. Adult, male Wistar rats (Charles River Laboratories, Wilmington, MA), weighing 250-300 g at the start of the experiment, were housed two per cage in a temperature-controlled vivarium under a reversed light/dark cycle (lights off 10:00 A.M. to 10:00 P.M.). Food and water were available ad libitum, except during the food training period. Rats were subjected to either methamphetamine self-administration (for experimental details, see Fig. 1a) or voluntary exercise on running wheels and were $24-26$ weeks old when perfused. All procedures were performed during the dark phase. During the study, all rats received one intraperitoneal injection of $150 \mathrm{mg} / \mathrm{kg}$ bromodeoxyuridine (BrdU) (Roche Diagnostics, Indianapolis, IN) (dissolved in $0.9 \%$ saline and $0.007 \mathrm{~N} \mathrm{NaOH}$ at $20 \mathrm{mg} / \mathrm{ml}$ ) and survived for $28 \mathrm{~d}$, after which they were anesthetized with chloral hydrate and perfused transcardially as described previously (Mandyam et al., 2004). Brains were sectioned coronally on a freezing microtome at $40 \mu \mathrm{m}$ through the mPFC (bregma 3.7-2.2) (Paxinos and Watson, 1997), and sections were stored in $0.1 \% \mathrm{NaN}_{3}$ in $1 \times \mathrm{PBS}$ at $4^{\circ} \mathrm{C}$. Surgical and experimental procedures were performed in strict adherence to the National Institutes of Health Guide for the Care and Use of Laboratory Animals (publication number 85-23, revised 1996) and approved by the Institutional Animal Care and Use Committee of The Scripps Research Institute.

Methamphetamine self-administration. Methamphetamine hydrochloride (generously provided by the National Institute on Drug Abuse, Bethesda, MD) was dissolved in sterile physiological saline (0.9\%). Methamphetamine self-administration was performed as reported previously (Kitamura et al., 2006). Details of the timeline of self-administration are provided in Figure 1a. Rats were food restricted from $1 \mathrm{~d}$ before the first day of food training, and food was given ad libitum after training. For baseline, all animals were allowed access to a fixed ratio 1 timeout $20 \mathrm{~s}$ schedule of methamphetamine self-administration with $1 \mathrm{~h}$ access to methamphetamine for six consecutive sessions at a dose of $0.05 \mathrm{mg} / \mathrm{kg}$ per infusion. After baseline, three groups of rats were formed and were subjected to escalation sessions. During the escalation phase, groups differed only with respect to methamphetamine availability: $6 \mathrm{~h}$ daily access [long access (LgA) group; $n=6$ ], $1 \mathrm{~h}$ daily access [short access (ShA) group; $n=6-9$ ], or $1 \mathrm{~h}$ intermittent access (I-ShA; $n=6$, exposed to methamphetamine on Monday and Thursday of the week). The selfadministration lasted for at least $21 \mathrm{~d}$ followed by BrdU injection and $28 \mathrm{~d}$ of survival. A parallel group of age-matched rats $(n=6)$ housed in the same room were used as drug-naive controls.

Voluntary exercise. After a $7 \mathrm{~d}$ acclimation period, each rat was randomly assigned to either standard caging (control) or caging that contained a running wheel (Nalgene activity wheel, $34.5 \mathrm{~cm}$ diameter $\times 9.7$ $\mathrm{cm}$ wide) connected to a Axiom A10W Cyclocomputer, allowing us to monitor running distance and speed in kilometers for individual animals. All voluntary exercise animals $(n=6)$ were exposed to the running wheel for $14 \mathrm{~d}$ to ensure running activity, after which they received a single intraperitoneal BrdU injection and were maintained with running wheels for $28 \mathrm{~d}$. Parallel groups of age-matched rats $(n=3)$ housed in the same room without running wheels were used as controls.

Antibodies. The following primary antibodies were used for immunohistochemistry: rabbit polyclonal anti-Ki-67 (1:500; Novocastra Laboratories, Newcastle upon Tyne, UK), mouse anti-BrdU (1:100; BD, Franklin Lakes, NJ), rat monoclonal anti-BrdU (1:100; Accurate Chemical, Westbury, NY), rabbit polyclonal anti-glial fibrillary acidic protein (GFAP; 1:500; DakoCytomation, High Wycombe, UK), mouse monoclonal anti-neuronal nuclear protein (NeuN; 1:50; Millipore, Billerica, $\mathrm{MA}$ ), and rabbit polyclonal anti-chondroitin sulfate proteoglycan NG2 (1:250; Millipore).

Immunohistochemistry. The left and right hemispheres of every ninth section through the rat brain mPFC were slide mounted, coded, and dried overnight before immunohistochemistry. Slide-mounted sections were subjected to three pretreatment steps as described previously (Mandyam et al., 2004). After pretreatments, sections were incubated with $0.3 \%$ hydrogen peroxide for $30 \mathrm{~min}$ to remove any endogenous peroxidase activity. Nonspecific binding was then blocked with $5 \%$ serum and $0.5 \%$ Triton X-100 in $1 \times$ PBS for 60 min and incubated with the primary antibody (in 5\% serum and $0.5 \%$ Tween 20 ) for $18-20 \mathrm{~h}$. After washing with $1 \times \mathrm{PBS}$, the sections were exposed to either biotin-tagged (single-labeling studies) or fluorescent-tagged (triple-labeling studies) secondary antibodies for $60 \mathrm{~min}$. For single labeling, after secondary antibody incubation, slides were incubated in $A B C$ for $1 \mathrm{~h}$ (Vector Laboratories, Burlingame, CA), and then staining was visualized with metalenhanced DAB substrate (Pierce, Rockford, IL). DAB-labeled sections were counterstained with fast red (Vector Laboratories). For triple labeling, the sections were simultaneously incubated with all primary antibodies (BrdU, NeuN, GFAP, or NG2), followed by sequential fluorescent secondary antibody staining [cyanine 2 (Cy2)-, Cy3-, and Cy5conjugated secondary antibodies (Jackson ImmunoResearch, West Grove, PA)]. Fluorescent-labeled sections were counterstained with 4',6diamidine-2' -phenylindole dihydrochloride (DAPI) (1:3000; Roche Diagnostics). Omission or dilution of the primary antibody resulted in a lack of specific staining, thus serving as a negative control for immunohistochemistry experiments.

Quantification, confocal imaging, and analysis. $\mathrm{mPFC}$ cells were quantified with a Zeiss (Oberkochen, Germany) Axiophot photomicroscope $(400 \times)$ using the optical fractionator method in which the left and right hemisphere of every ninth section through the mPFC (bregma 3.7-2.2) was examined. Cells from each bregma region were summed and multiplied by nine to give the total number of cells.

All BrdU-immunoreactive (IR) cells were subjected to phenotypic analysis with a confocal microscope [LaserSharp 2000, version 5.2 (BioRad, Hercules, CA); emission wavelengths, 488, 568, and $647 \mathrm{~nm}]$. The ratio of BrdU-IR cells that were BrdU/NeuN, BrdU/GFAP, BrdU/NG2, or BrdU-IR cells negative for NeuN, NG2, and GFAP in relation to the total number of BrdU cells were analyzed from each rat. The proportion of BrdU/NeuN-IR cells from both BrdU/NeuN/GFAP and BrdU/NeuN/ NG2 combinations was combined for each rat. Standards for BrdU/ NeuN assessment included 100\% colocalization of BrdU-IR cell with $\mathrm{NeuN}$ and equal penetration and labeling of both fluorescent dyes when visualized under orthogonal scanning. Standards for BrdU/GFAP assessment included GFAP staining surrounding BrdU-IR cell to be $>80 \%$ and equal penetration and labeling of both fluorescent dyes when visualized under orthogonal scanning. Standards for BrdU/NG2 assessment included $100 \%$ colocalization of BrdU-IR with cell body of NG2-IR cell and equal penetration and labeling of both fluorescent dyes when visualized under orthogonal scanning.

Optical sectioning in the $z$ plane was performed using multitrack scanning with an optimal section thickness of $0.45 \mu \mathrm{m}$. Confocal analysis was performed at $600 \times$ and restricted to the top $15 \mu \mathrm{m}$ of the section where penetration of all three antibodies is reliable. Cells in the uppermost range of a section were omitted from analysis to avoid counting end caps. Colocalization of antibodies was assessed with the confocal system by analysis of adjacent $z$ sections (gallery function) and orthogonal sectioning $(x-y-z$ plane) through single $z$ sections. Three-dimensional render- 
ings were rotated, and colocalization was examined from $x-, y$-, and $z$-axes.

Stereology volume analysis. Volume measurements were determined in the sections used previously to measure proliferation counterstained with fast red. Volumes were estimated based on surface area measurements made from coronal brain sections. All measurements were obtained using StereoInvestigator software (MicroBrightField, Williston, VT) with a Zeiss Axiophot photomicroscope. Outlines were traced around the border of the $\mathrm{mPFC}$ at low power $(50 \times)$ in every ninth section from rostral to caudal throughout the $\mathrm{mPFC}$ (four sections bilaterally). All contours were drawn using a $5 \times$ objective, and a grid spacing of $150 \mu \mathrm{m}$ was used to determine the $x$ and $y$ spacing between neighboring points in the array. A randomized rotation was used for superimposing the grid on the contours to perform volume estimates according to the Cavalieri principle. Sections outlined for mPFC included cingulate cortex areas 1 and 3, infralimbic cortex, and prelimbic cortex. The number of sampling sites ranged from 85 to 150 (bregma 3.7-2.2) in the mPFC. A section thickness of $40 \mu \mathrm{m}$ and measured section thickness of $21 \mu \mathrm{m}$ were used for all groups.

Data analysis. Data are represented as mean \pm SEM and are considered significant if $p<0.05$. The relationship between methamphetamine intake Ki-67, BrdU, and pyknotic cells and voluntary exercise Ki-67 and BrdU was determined using Pearson's regression analyses. Statistical analysis was performed with either one- or two-way ANOVA followed by Dunnett's or Bonferroni's post hoc test or Student's $t$ test using GraphPad Software (San Diego, CA) Prism version 4.03 for Windows. Images presented here were collected on a Bio-Rad LaserSharp 2000 version 5.2 confocal microscope and imported into Photoshop version CS2 (Adobe Software, San Jose, CA). Only the gamma adjustment in the levels function was used.

\section{Results}

Methamphetamine decreases proliferation and survival of mPFC precursors

Self-administration behavior

We used the limited and daily access model of intravenous methamphetamine self-administration to investigate methamphetamine-induced alterations on actively proliferating cells and survival of precursors in the mPFC. Rats were implanted with catheters for methamphetamine self-administration in the jugular vein as described previously (Kitamura et al., 2006). After baseline self-administration was achieved, rats were placed in one of three groups of methamphetamine exposure: intermittent $1 \mathrm{~h}$ (I-ShA), daily $1 \mathrm{~h}$ (ShA), or $6 \mathrm{~h}$ access (LgA) as described in Materials and Methods (Fig. 1). The three groups did not show any differences in baseline methamphetamine intake (Fig. 1b,c, Baseline). After extended access to self-administration, there was a significant interaction between the three groups (Fig. 1b) $\left(F_{(4,36)}\right.$ $=9.7 ; p<0.0001)$, indicating differences in drug intake. During the first hour access of an earlier session (day 19) and the last session (day 49), the LgA group showed a significant increase in methamphetamine intake compared with baseline. By the last session (day 49), the LgA group also showed a significant increase in methamphetamine intake compared with day 19 (Fig. 1b). A significant increase in intake also was observed between the last session (day 49) and baseline in the ShA group (106\%) but to a lesser extent compared with the LgA group (262\%) (Fig. 1b,c). No increase in intake was observed in the I-ShA group (Fig. 1b,c). During the first hour access of the last session (day 49), the LgA group showed a significant increase in methamphetamine intake compared with the ShA and I-ShA groups, and the ShA group showed an increase in intake compared with the I-ShA group. Therefore, the self-administration data from the I-ShA, ShA, and LgA groups provide evidence for distinct patterns of methamphetamine intake that may be related to human patterns of use: recreational, chronic abuse, and dependence. The distinct set of
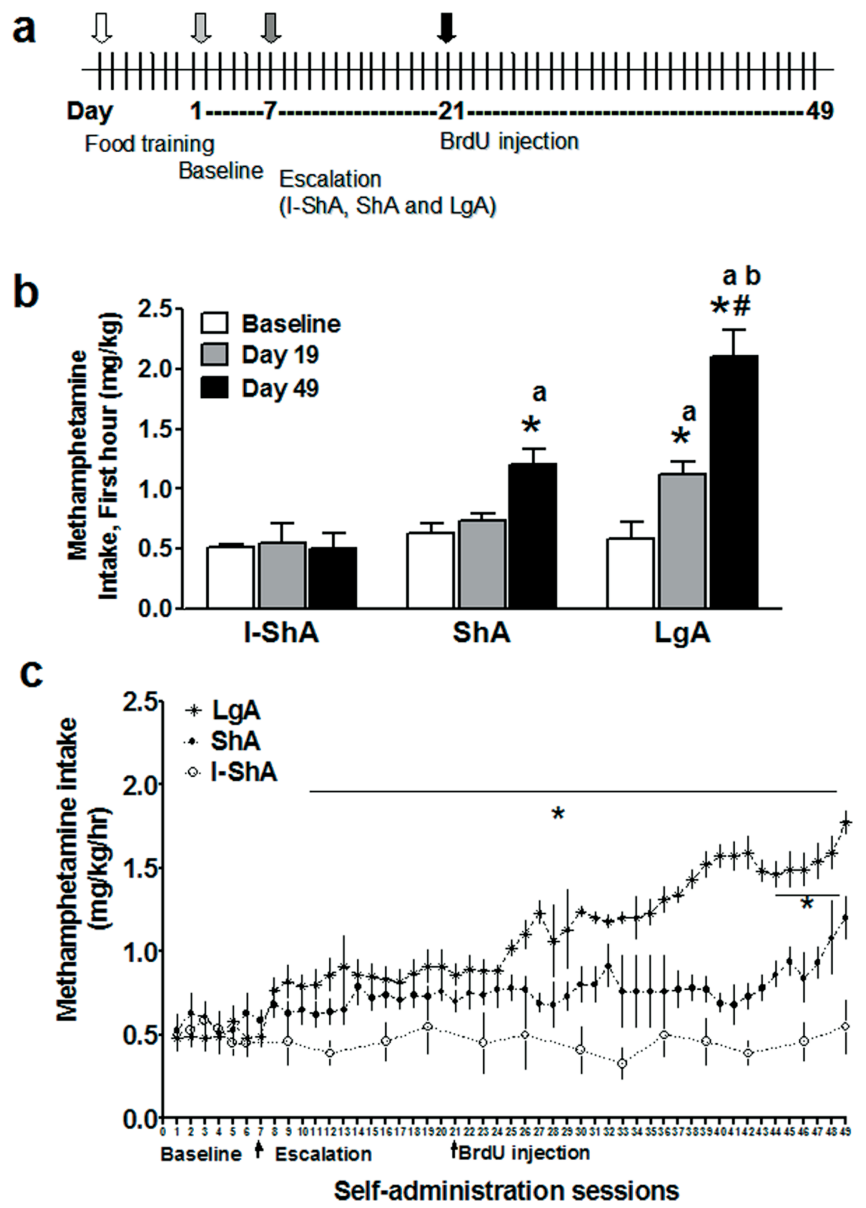

Figure 1. Experimental design and escalation of methamphetamine self-administration. $\boldsymbol{a}$, Timeline of methamphetamine self-administration and BrdU injection. Each vertical line represents $1 \mathrm{~d}$. The entire self-administration experiment lasted for $49 \mathrm{~d}$. $\boldsymbol{b}$, Effect of daily short or extended access ( $1 \mathrm{~h} \mathrm{ShA;} n=6)$ and $(6 \mathrm{~h} \mathrm{LgA} ; n=6)$ versus intermittent short access $(1 \mathrm{~h}$ I-ShA; $n=6$ ) to methamphetamine self-administration on methamphetamine intake during the first hour of self-administration. LgA increased methamphetamine intake on days 19 and 49 compared with baseline (days 1-7 combined), and day 49 showed an increase in intake compared with day 19. ShA increased methamphetamine intake on day 49 compared with baseline. LgA (day 49) showed an increase in intake compared with I-ShA and ShA. ShA also showed an increase in intake compared with I-ShA. c, Methamphetamine intake per hour from I-ShA (1 h), ShA ( $1 \mathrm{~h})$, and LgA (6h) groups. * $p<0.05$ compared with baseline; ${ }^{*} p<0.05$ compared with day 19 in $\boldsymbol{b}$ and compared with days $7-10$ in $\boldsymbol{c} ;{ }^{a} p<0.05$ compared with the same day in I-ShA; ${ }^{b} p<0.05$ compared with same day in ShA by Tukey's post hoc test. Data are represented as mean \pm SEM.

symptoms of methamphetamine intake presents a useful model to explore methamphetamine dependence-induced alterations in adult brain plasticity.

\section{Histology}

Brains from the drug-naive control, I-ShA, ShA, and LgA groups were processed and examined for changes in precursor proliferation (Ki-67-IR), survival (BrdU-IR), and cell death (pyknotic cells) in the mPFC. For immunoreactive cell quantification, all mPFC subregions [anterior cingulate cortex (ACC), prelimbic cortex, and infralimbic cortex] were included in four rostrocaudal levels of the mPFC (360 $\mu \mathrm{m}$ apart; bregma 3.7, 3.2, 2.7, and 2.2) (Fig. $2 a-c, g$ ). The number of cells from each bregma region and the total number of cells for the entire mPFC (threedimensional area per bilateral mPFC) are indicated in Figure 2. 

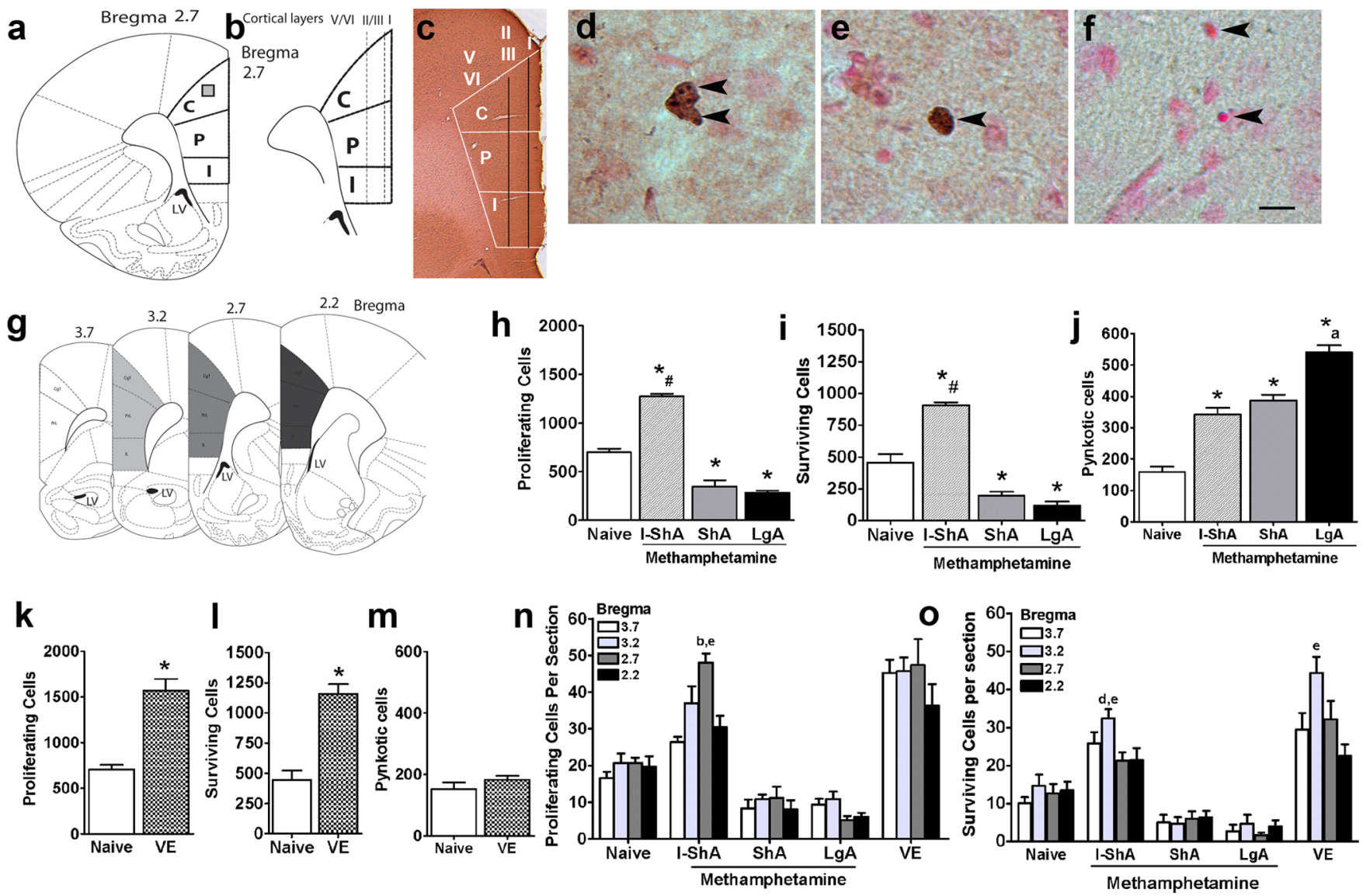

Figure 2. Effect of methamphetamine self-administration and voluntary exercise on cell proliferation, cell survival, and cell death in the mPFC. $\boldsymbol{a}$, Schematic representation of the mPFC subregions (bregma 2.7) analyzed for cell quantification. Subregions included are anterior cingulate (C), prelimbic (P), and infralimbic (I) cortices. The boxin C-subregion of the $\mathrm{mPFC}$ denotes the area for pictures shown in $\boldsymbol{d}$-f. $\boldsymbol{b}$, Schematic of the mPFC with cortical layers (Stewart and Plenz, 2006). $\boldsymbol{c}$, Magnified image (25X) of bregma 2.7 stained for Ki-67 and counterstained with fast red. The areas examined (mPFC with subregions and cortical layer distribution) are included. $\boldsymbol{d}-\boldsymbol{f}$, DAB-stained sections showing immunoreactive cells labeled with Ki-67 (d), BrdU (e), and fast red-stained section showing pyknotic cells $(\boldsymbol{f})$ in the anterior cingulate of the mPFC. $\boldsymbol{g}$, Schematic of all bregma regions included for quantification. $\boldsymbol{h}$-j, Quantitative analysis of total number of cells for cell proliferation (Ki-67; $\boldsymbol{h}$ ), cell survival (BrdU; $\boldsymbol{i})$, and cell death (pyknotic cells; $\boldsymbol{j}$ ) after methamphetamine self-administration. $\boldsymbol{k}-\boldsymbol{m}$, Quantitative analysis of total number of cells for cell proliferation (Ki-67; $\boldsymbol{k}$ ), cell survival (BrdU; $I)$, and cell death (pyknotic cells; $\boldsymbol{m}$ ) after voluntary exercise (VE). $\boldsymbol{n}, \mathbf{0}$, Quantitative analysis indicated as cells per section for cell proliferation (Ki-67; $\boldsymbol{n}$ ) and cell survival $(\mathrm{BrdU} ; \boldsymbol{0}) . n=6-9$ in each group, ${ }^{*} p<0.05$ versus control by Dunnett's posthoc analysis; ${ }^{* \#} p<0.05$ versus control and daily access groups; ${ }^{*} P<0.05$ versus control, I-ShA, and ShA groups; ${ }^{b} p<$ 0.05 versus bregma 3.7 within each group; ${ }^{d} p<0.05$ versus bregma 2.7 within each group; ${ }^{e} p<0.05$ versus bregma 2.2 within each group by Tukey's post hoc test. Scale bar: (in $\boldsymbol{f}$ ) $\boldsymbol{d}-\boldsymbol{f}, 10 \mu \mathrm{m}$. Data are represented as mean \pm SEM.

\section{Cell proliferation in the $m P F C$}

Precursor proliferation was evaluated by Ki-67 immunohistochemistry (Fig. 2d), an endogenous marker specific for actively dividing precursors (Bacchi and Gown, 1993). Daily access to methamphetamine (ShA and LgA groups) decreased Ki-67 cell counts compared with controls (Fig. $2 h, n)\left(F_{(3,25)}=86.07 ; p<\right.$ 0.0001 ), and the decrease was uniform in the bregma regions analyzed. Intermittent access to methamphetamine (I-ShA group) substantially increased Ki-67 cell counts compared with control and daily access groups (Fig. $2 h, n)\left(F_{(3,25)}=86.07 ; p<\right.$ $0.0001)$, and the increased proliferation was higher in bregma region 2.7 (Fig. $2 n)\left(F_{(3,25)}=9.2 ; p<0.001\right)$.

Cell survival in the $\mathrm{mPFC}$

BrdU immunohistochemistry was performed to quantify 28-dold BrdU-IR cells (Fig. 2e), a measure of precursor cell survival (Corotto et al., 1993). Parallel to the observed changes in cell proliferation, daily access to methamphetamine (LgA and ShA groups) significantly decreased BrdU cell counts compared with controls, and intermittent exposure to methamphetamine (IShA group) increased the number of mature BrdU cells compared with control and extended access groups (Fig. $2 i, o)\left(F_{(3,16)}\right.$
$=42.29 ; p<0.001)$. The most robust effect in the I-ShA group was seen in bregma region 3.2 (Fig. $2 o)\left(F_{(3,16)}=3.67 ; p<0.05\right)$, suggesting that the increase in mature cells was not equivalent at every level of the mPFC (Fig. 2o).

\section{Cell death in the $m P F C$}

Pyknotic cells (Fig. $2 f$ ) were quantified to assess changes in cell death. I-ShA, ShA, and LgA groups showed increased numbers of pyknotic cells in the mPFC compared with controls (Fig. 2j) $\left(F_{(3,16)}=54.49 ; p<0.001\right)$. Importantly, the LgA group showed more cell death in the mPFC than the I-ShA and ShA groups (Fig. 2l) $\left(F_{(3,16)}=54.49 ; p<0.006\right)$.

Simple regression analysis demonstrated a negative correlation between methamphetamine intake, proliferation, and survival of mPFC precursors (Pearson's correlation; Ki-67, $r=$ $-0.69, r^{2}=0.46, p=0.002$; BrdU, $r=-0.80, r^{2}=0.64, p=$ $0.001)$ and a positive correlation between methamphetamine intake and cell death (pyknotic cells, $r=0.91, r^{2}=0.84, p<0.001$ ).

\section{Volume of the $m P F C$}

Previous reports have implied that chronic stress decreased hippocampal volume by decreasing hippocampal proliferation 
(Czeh et al., 2001). Therefore, the contribution of structural changes in the mPFC to decreases in precursor cell birth and maturity after exposure to methamphetamine in ShA and LgA groups was explored. Daily access to methamphetamine (ShA and LgA groups) did not alter the volume of the mPFC (right and left hemisphere combined $\mathrm{mPFC}$ volume: naïve, $9.2 \pm 0.3 \mathrm{~mm}^{3}$; ShA, $8.5 \pm 0.5 \mathrm{~mm}^{3}$; LgA, $\left.8.8 \pm 0.3 \mathrm{~mm}^{3} ; p=0.45\right)$ despite decreases in proliferation and survival of $\mathrm{mPFC}$ precursors.

\section{Voluntary exercise increases proliferation and survival of mPFC precursors}

Training

Rats were given free access to running wheels in their home cage $24 \mathrm{~h} / \mathrm{d}$. After 2 weeks of acclimation to the environment, running activity was monitored. The average running distance per day varied between individual rats (low of $0.17 \pm 0.01 \mathrm{~km} / \mathrm{d}$ to a high of $1.01 \pm 0.1 \mathrm{~km} / \mathrm{d}$ ), which was maintained until the end of the experiment. It is important to note that rats in the voluntary exercise group did not gain as much weight as the control group (percentage weight gain over the entire experimental period: control, $22.7 \pm 0.08$ vs voluntary exercise, $14.0 \pm 1.3 ; p<0.05$, unpaired $t$ test).

\section{Histology}

Brains from the control and voluntary exercise groups were processed for Ki-67, BrdU, and pyknotic cell staining. Voluntary exercise increased Ki-67 cell counts in the mPFC compared with controls (Fig. $2 k, n, \mathrm{VE})(p=0.002)$, and the increase in proliferation was seen in all bregma regions analyzed. Exercise also increased mature BrdU cell counts compared with controls (Fig. $2 l, o, \mathrm{VE})(p=0.001)$, and, unlike proliferation, bregma region 3.2 showed the most robust increase (Fig. $20, \mathrm{VE})\left(F_{(3,23)}=4.7\right.$; $p<0.01)$. Exercise did not alter levels of cell death compared with controls. Because there was variability in running activity between rats, a possible correlation between the amount of exercise and cell genesis was evaluated. Simple regression analysis showed no correlation between running activity, proliferation, and survival of mPFC precursors (Ki-67, $r^{2}=0.19, p=0.38$; BrdU, $r^{2}=$ $0.23, p=0.32$ ).

\section{Mature cell phenotype in the mPFC is mainly gliogenic, but also to a lesser extent neurogenic}

Four weeks after BrdU injection, BrdU-IR cells in the mPFC from all groups were subjected to phenotypic analysis. These included controls (drug-naive, controls from the methamphetamine group, and exercise naive from voluntary exercise group), methamphetamine-exposed (I-ShA, ShA, and LgA), and voluntary exercise. Drug-naive controls from the methamphetamine study and controls from the voluntary exercise study showed similar levels of BrdU-IR cell counts; therefore, all of the controls were pooled for analysis (BrdU-IR cells: drug-naive controls, $471 \pm 78, n=6$ vs voluntary exercise controls, $444 \pm 125, n=3$; $p=0.86$ by unpaired $t$ test). BrdU-IR cells were triple labeled with markers for neurons (NeuN), astrocytes (GFAP), and oligodendrocytes (chondroitin sulfate proteoglycan NG2). Immunohistochemistry for BrdU/NeuN/GFAP or BrdU/NeuN/NG2 was performed, and the percentage of BrdU-IR cells that colocalized with neurons versus astrocytes or neurons versus oligodendrocyte precursors was determined.

From the naive control group, every BrdU-IR cell in the $\mathrm{mPFC}$ (mean \pm SEM, $37 \pm 7$ BrdU-IR cells from each rat) was subjected to confocal microscopy analysis, including optical sectioning in the $z$ plane, orthogonal analysis, and three-dimensional recon- struction. Approximately 35\% of mPFC BrdU-IR cells colocalized with GFAP (Fig. $3 d 1-d 4, g 3$ ) [>80\% colabeling of BrdU with GFAP, overall density of astrocytes of $19 \pm 3.8 \mathrm{BrdU} / \mathrm{GFAP}$ cells/ $\mathrm{mm}^{3}$; density of cells were estimated as total number of BrdU/ GFAP-IR cells in the mPFC $(172.5 \pm 32.2)$ (Fig. 3i) divided by estimated volume of $\mathrm{mPFC}(9.2 \pm 0.3)]$, and $55 \%$ of $\mathrm{mPFC}$ BrdU-IR cells colocalized with NG2 (Fig. 3e,f1-f4) (100\% colabeling of BrdU with NG2-IR cell body, overall density of oligodendrocytes of $27.8 \pm 3.6 \mathrm{BrdU} / \mathrm{NG} 2$ cells $/ \mathrm{mm}^{3}$ ). These data support previous work that most cortical precursors in the adult mPFC become glia (Gould et al., 1999b; Magavi et al., 2000; Nowakowski and Hayes, 2000; Kornack and Rakic, 2001; Bernier et al., 2002; Ehninger and Kempermann, 2003; Koketsu et al., 2003; Kodama et al., 2004; Dayer et al., 2005; Madsen et al., 2005; Czeh et al., 2007; Ongur et al., 2007). BrdU-IR/GFAP-IR and BrdU-IR/NG2-IR cells were evenly distributed in all layers of the $\mathrm{mPFC}$. Interestingly, of the nine naive rat brains analyzed, eight showed BrdU-IR/NeuN-IR cells in the mPFC, suggesting these cells achieved a neuronal phenotype (Fig. $3 a, c 1-c 4, g 4,1, m 1-m 3$ ) ( $100 \%$ colabeling of BrdU with NeuN). In these eight rats, $5.6 \pm$ $1 \%$ of mPFC BrdU-IR cells colocalized with NeuN, indicating the overall density of BrdU/NeuN-IR cells to be $2.6 \pm 0.4$ cells $/ \mathrm{mm}^{3}$. All BrdU-IR/NeuN-IR cells were in layers V/VI of the cingulate cortex (Fig. 2a-c) (cortical layer distribution adopted from Stewart and Plenz, 2006), with none in prelimbic or infralimbic structures. In all control rats, in addition to the large population of BrdU glial cells (Fig. 3j,k), and smaller but significant population of BrdU neuronal cells, a small proportion of the BrdU-IR cells (1-3\%) (Fig. $3 g 1, g 2$ ) did not colocalize with NeuN, GFAP, or NG2.

Phenotypic analysis of mature BrdU-IR cells in the mPFC from the I-ShA, ShA, and LgA groups revealed significant changes in the phenotypic ratio. Every BrdU-IR cell in the mPFC (BrdU-IR cells, mean \pm SEM: I-ShA, $63 \pm$ 9; ShA, $26 \pm 4$; LgA, $15 \pm 7$ ) was subjected to confocal $z$ scanning. The methamphetamine groups showed similar ratios of BrdU/NeuN colabeling compared with controls (Fig. 3h) (ratio of BrdU/NeuN cells: control, $5.6 \pm 1$; I-ShA, $3.6 \pm 1.1 ; \operatorname{ShA}, 3.8 \pm 0.7 ; \operatorname{LgA}, 3.9 \pm 1.4 ; p>$ $0.05)$. Therefore, the total number of BrdU-IR cells that matured into a neuronal phenotype (NeuN-IR) was maintained after intermittent methamphetamine self-administration and was decreased in the ShA and LgA groups (Fig. 3i) $\left(F_{(4,27)}=3.6 ; p=\right.$ 0.012 ). Intermittent access to methamphetamine (I-ShA) decreased the proportion of BrdU-IR cells that matured into astrocytes compared with controls (Fig. $3 h)\left(F_{(4,27)}=2.7 ; p=0.042\right)$ and therefore did not change the total number of BrdU/GFAP-IR cells (Fig. 3j). Both ShA and LgA groups decreased the total number of BrdU/GFAP-IR cells (Fig. $3 j)\left(F_{(4,27)}=12.2 ; p=0.001\right)$. In contrast to the effects on neurons and astrocytes, daily extended access to methamphetamine (LgA) decreased the proportion of BrdU-IR cells that matured into oligodendrocyte precursors compared with controls (Fig. $3 h)\left(F_{(4,27)}=2.5 ; p=0.039\right)$. I-ShA increased, whereas ShA and LgA decreased, the total number of BrdU/NG2-IR cells (Fig. $3 k)\left(F_{(4,27)}=28.4 ; p=0.001\right)$. In the voluntary exercise group, every BrdU-IR cell in the mPFC (mean \pm SEM, $72 \pm 7$ BrdU-IR cells from each rat) were subjected to similar phenotypic analysis. Exercise maintained and did not alter the phenotypic ratio of mature BrdU-IR cells in the mPFC (Fig. 3h, VE). Importantly, voluntary exercise increased the total number of BrdU/GFAP-IR (Fig. $3 j)\left(F_{(4,27)}=12.2 ; p=\right.$ $0.001)$ and BrdU/NG2-IR cells (Fig. $3 k)\left(F_{(4,27)}=2.5 ; p=0.039\right)$, without altering the total number of BrdU/NeuN-IR cells. 


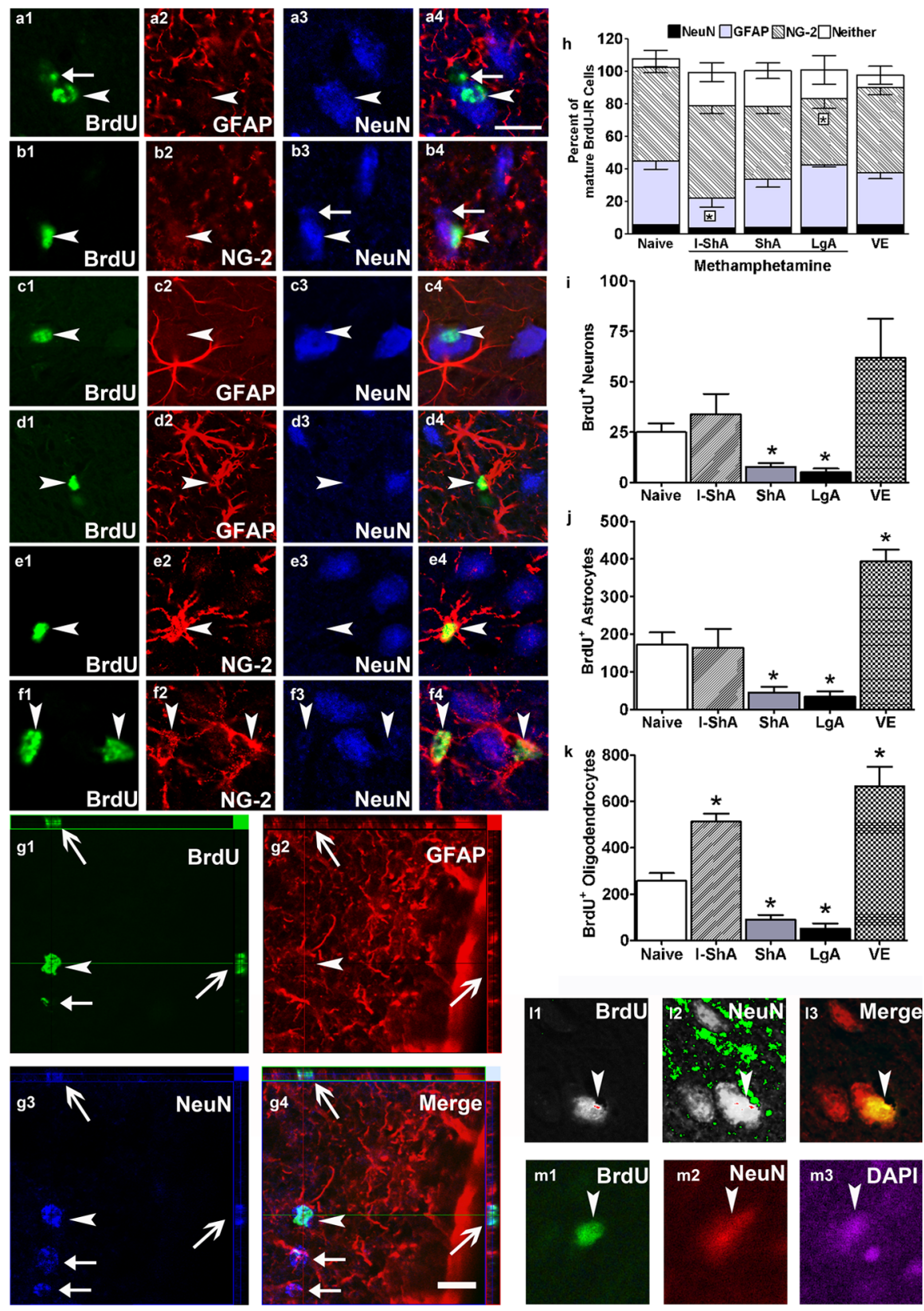

Figure 3. Phenotypic analysis of surviving BrdU-IR cells in the mPFC. Single $z$ plane $(0.45 \mu \mathrm{m}$ thick) images of BrdU-labeled

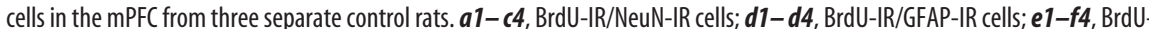
IR/NG2-IR cells. BrdU-IR cells that were close to or touching NeuN-IR cells were mostly NG2-IR (as shown in f1-f4). BrdU is in Cy2 (green), NeuN is in Cy5 (blue), and GFAP and NG2 are in Cy3 (red). To further support NeuN/BrdU labeling (g1- g4) orthogonal sections from one BrdU cell are included. The orthogonal sectioning provides images of the labeled cell in the three dimensional $(x-y-z)$ angle supporting labeling of entire cell and not overlapping or touching cells. g1-g4, Arrowhead points to BrdU/NeuN-IR cell in $z$ angle; open arrows point to the same cell [positive for BrdU $(\boldsymbol{g} \mathbf{1})$ and NeuN $(\boldsymbol{g} 3)$ and negative for GFAP $(\boldsymbol{g} 2)$ in $x$ and $y$ angle]. Filled arrows in $\boldsymbol{g} \mathbf{1 - g} \mathbf{4}$ point to $\mathrm{NeuN}^{+} / \mathrm{BrdU}$-negative $\left(\mathrm{BrdU}^{-}\right.$) cells. Confocal images of one BrdU/NeuN/DAPIIIR cell shown in black and white $(\mathbf{I 1}, \mathbf{I})$ and color (I3) to demonstrate optimal exposure of each fluorescent marker (II, BrdU-Cy2; I2 $\mathrm{NeuN}-(\mathrm{y} 3)$, avoiding overexposure or underexposure that may produce false-positive colabeling and to show that the BrdUlabeled cell colocalizes with the NeuN-labeled cell via a confocal $z$ image. Epifluorescent images ( $\boldsymbol{m} 1-\boldsymbol{m} \mathbf{3})$ of the BrdU cell shown in $\mathbf{Z}$; BrdU-labeled cell has only one DAPI-labeled nucleus $(\boldsymbol{m} \mathbf{3})$. Arrowhead in each image points to an immunoreactive cell positive for BrdU/NeuN (a1- $\mathbf{c}$ ), BrdU/GFAP (d1- $\mathbf{d 4}$ ), and BrdU/NG2 (e1-f4). Filled arrow in $\boldsymbol{a} 1$ and $\boldsymbol{a} 4$ points to a BrdU ${ }^{+} /$ $\mathrm{NeuN}^{-}$cell; filled arrow in $\boldsymbol{b} \mathbf{3}, \boldsymbol{b 4}, \boldsymbol{g} \mathbf{3}$, and $\boldsymbol{g} \mathbf{4}$ points to $\mathrm{NeuN}^{+} / \mathrm{BrdU}^{-}$cell. $\boldsymbol{h}$, Qualitative analysis of BrdU-IR cells from control, voluntary exercise, and methamphetamine-exposed groups. $n=4-9$ in each group, ${ }^{*} p<0.05$ versus control by Dunnett's post hoc analysis. $\boldsymbol{i}-\boldsymbol{k}$, Quantitative data demonstrating the effect of methamphetamine self-administration (I-ShA, ShA, and LgA) and voluntary exercise (VE) on total number of BrdU-IR cells that were colabeled with NeuN (i), GFAP (i), or NG2 (k). Scale bars: (in a4) a1-f4, I1-m3; (in g4) $g$ 1-g4, $10 \mu \mathrm{m}$. Qualitative analysis is expressed as mean \pm SEM.

\section{Discussion}

Methamphetamine abuse results in structural, morphological, and physiological injury to the PFC (Paulus et al., 2002; Prudencio et al., 2002; Sekine et al., 2003; Kadota and Kadota, 2004; Lehmann et al., 2004; Thompson et al., 2004) and, as shown in the present study, decreased adult $\mathrm{mPFC}$ gliogenesis. Given that the $\mathrm{mPFC}$ is implicated in drug craving and seeking (Jentsch et al., 2000; Rhodes et al., 2005) and that glial cells can have a profound impact on brain function (Bartzokis et al., 2004), our data emphasize that decreased mPFC gliogenesis should be considered as a potent contributor to the compulsive drug-seeking and frontal cortical behavioral deficits associated with psychostimulants.

The findings of this study demonstrate the dynamic regulation of adult mPFC proliferative capacity and underscore how methamphetamine self-administration differentially influences cell birth, survival, and death relative to the amount of drug exposure. For example, intermittent short access (I-ShA; "recreational" or "weekend abuse") to methamphetamine did not produce a dependence-like syndrome but increased mPFC proliferation, survival (oligodendrocytes), and cell death. These results suggest additional investigation into whether the intermittent methamphetamine-induced enhancement in proliferation and gliogenesis contributes to the transient increase in alertness and wakefulness observed in humans with recreational use of methamphetamine (Barr et al., 2006). Unlike intermittent access, daily short and extended access to methamphetamine (ShA and LgA) generated a dependence-like syndrome and decreased mPFC proliferation and survival (neurogenesis and gliogenesis) and increased cell death. These changes reveal possible mechanisms underlying the functional neurotoxic effects seen with methamphetamine dependence (Paulus et al., 2002; Prudencio et al., 2002; Sekine et al., 2003; Kadota and Kadota, 2004; Lehmann et al., 2004; Thompson et al., 2004). Furthermore, the negative correlation of methamphetamine intake and precursor proliferation and survival, and the positive correlation of methamphetamine intake and cell death, underscore the harmful effects of chronic methamphetamine use on mPFC neuroplasticity. Perhaps even more importantly, the present results show how even limited exposure to a powerful drug of abuse, such as methamphetamine, can initiate changes in mPFC cell proliferation, survival, and cell death. 
Having demonstrated the disruptive effects of methamphetamine dependence on mPFC gliogenesis, the outcome of voluntary exercise [a stimulus known to be proneurogenic in the hippocampal subgranular zone (van Praag et al., 1999)] on mPFC plasticity was explored for comparison. Only a few additional studies have reported a positive influence of voluntary exercise on cortical cytoarchitecture and function. For example, voluntary exercise increases vasculature in adult rat motor cortex (Kleim et al., 2002) and improves function dependent on the frontal cortex in normal and cognition-impaired patients (Pinilla, 2006; Small et al., 2006; Yu et al., 2006). Interestingly, voluntary exercise did not alter gliogenesis in the adult mouse neocortex but rather increased proliferation and survival of microglia in the ACC of the mPFC (Ehninger and Kempermann, 2003). However, given that the ACC is only one portion of the larger mPFC and because the adult cortex possesses endogenous neural precursors (Magavi et al., 2000; Hoehn et al., 2005), species differences may contribute to the neurogenic differences between the adult rat and mouse. Therefore, studies examining the effects of voluntary exercise on proliferation and survival in the adult rat mPFC were warranted.

Voluntary exercise, regardless of the amount of exercise, did not alter neurogenesis but enhanced $\mathrm{mPFC}$ gliogenesis (astrocytes and oligodendrocytes), actually more so than intermittent limited access to methamphetamine. Notably, running activity did not correlate with the amount of proliferation and survival in the $\mathrm{mPFC}$, suggesting that the amount of exercise per se may be less important than the difference between exercise and no exercise on mPFC plasticity. In addition, voluntary exercise enhanced gliogenesis without influencing cell death or mature phenotype, underscoring the distinct regulation of $\mathrm{MPFC}$ precursors and cell death by exercise versus methamphetamine. Furthermore, it is possible that the underlying mechanisms contributing to the proproliferative environment (increased cell proliferation and gliogenesis by I-ShA and voluntary exercise) are different. For example, the I-ShA-induced proliferative environment is perhaps attributable to changes in endogenous levels of neurotransmitters in the mPFC, especially dopamine, in which activation of distinct dopamine receptors have been pro-proliferative (Van Kampen et al., 2004). The voluntary exercise-induced proproliferative environment is possibly attributable to changes in vasculature and increased expression of endogenous growth factors that promote proliferation (Olson et al., 2006). Such mechanistic differences may contribute to the differential regulation of astrocytes versus oligodendrocytes seen by exercise versus intermittent methamphetamine.

Most rodent neocortical neurons are generated during development (Bayer and Altman 1990). Although controversial, some adult-generated NeuN-IR cells (putatively new neurons) are generated in the adult neocortex, including the PFC (Gould et al., 1999b; Bernier et al., 2002; Dayer et al., 2005). Despite several reports to the contrary (Kornack and Rakic, 2001; Madsen et al., 2005), our data support the hypothesis that the adult neocortex provides a neurogenic environment induced by external factors (Magavi et al., 2000) and gives birth to oligodendrocyte precursors (Kornack and Rakic, 2001; Kodama et al., 2004; Madsen et al., 2005, Czeh et al., 2007), which can be reprogrammed to become neural stem cells (Kondo and Raff, 2000). Therefore, it is intriguing to consider that the adult PFC may in fact support neurogenesis of cortical precursors whose phenotype is reported to be mostly GABA-positive interneurons (Dayer et al., 2005). To test this hypothesis, the identity of mature BrdU-IR cells in the mPFC was characterized. NeuN was used to mark mature neu- rons, because it is a transcription factor expressed in the nucleus and cytoplasm of mature neurons and is widely accepted to be neuron specific. Enhanced immunohistochemical analysis with detailed confocal microscopy analysis revealed a very small proportion of BrdU-IR cells that were NeuN-IR (3-5\%; $2.6 \pm 0.4$ BrdU/NeuN-IR cells $/ \mathrm{mm}^{3}$ ). The present study used nontoxic doses of BrdU (Cameron and McKay, 2001) to label S-phase cells, and no evidence was found of mPFC BrdU-IR/NeuN-IR cells undergoing cell death. This suggests that neurons were generated in the adult male Wistar rat $\mathrm{mPFC}$, at a similar number previously reported in nonhuman primates (Gould et al., 1999b) and adult male Sprague Dawley rat cortices (Dayer et al., 2005). Of the nine control rats, eight showed BrdU/NeuN-IR cells in the mPFC. The lack of BrdU/NeuN-IR cells in one rat's mPFC sections is possibly attributable to genetic, environmental, or other confounding variables influencing rates of neurogenesis that are supported by other studies (Dayer et al., 2005).

Alternatively, other confounding factors, such as NeuN labeling non-neuronal cells (Mullen et al., 1992) or oligodendrocyte precursors expressing lower levels of NeuN may negate the neuronal characteristics in the very small number of BrdU-IR/ NeuN-IR cells seen in our sections. There have been several recent reports that have examined in detail the phenotype of either all BrdU-IR cells in the adult rat mPFC ( similar to our analysis) or randomly selected 50 or more BrdU-IR cells in the mPFC, but none have reported BrdU-IR/NeuN-IR cells (Kornack and Rakic, 2001; Kodama et al., 2004; Madsen et al., 2005; Czeh et al., 2007; Ongur et al., 2007). Because our analysis shows very few BrdUIR/NeuN-IR cells $(2.6 \pm 0.4$ cells per cubic millimeter of PFC, restricted to only the layers V/VI of the anterior cingulate cortex), the probability of missing these cells (3-5\%) may be a factor for phenotypic differences between laboratories. Additionally, the use of Wistar rats in our study may add to the reports of phenotypic differences reported with the commonly used Sprague Dawley rats. Other evidence such as colabeling of oligodendrocyte precursor marker NG2 with mature BrdU-IR cells further supports the neurogenic environment in the mPFC (Dayer et al., 2005; Czeh et al., 2007; Ongur et al., 2007). Despite strong evidence that supports NG2 labeling in precursors that mature into oligodendrocytes (Dawson et al., 2003; Lin et al., 2006), recent evidence also supports that all NG2-IR cells do not mature into oligodendrocytes but rather generate functional neurons as well (Belachew et al., 2003; Aguirre et al., 2004; Dayer et al., 2005; Sellers and Horner, 2005). Additional markers are therefore necessary to determine the mature fate of BrdU/NG2-IR cells. Nonetheless, in the present study, all treatment groups (both voluntary exercise and methamphetamine-exposed) showed a high percentage of BrdU/NG2 colabeling and nominal levels of BrdU/ NeuN-IR cells (presumably GABAergic interneurons generated from NG2-IR cells) compared with controls, suggesting that BrdU/NeuN-IR cells, per se, did not significantly contribute to the behavioral effects or altered mPFC plasticity.

Unlike generation of new neurons that are restricted to development, there is considerable output of new glia in the adult mPFC. Here, functional implications for mPFC gliogenesis are reported, because physical activity and intermittent exposure to the psychostimulant increased gliogenesis. Both factors increased oligodendrocytes, a cell type that makes up the critical components of white matter microstructure, therefore suggesting possible improvements in working memory dependent on the PFC (Olesen et al., 2003). Daily methamphetamine exposure decreased both astrocytes and oligodendrocytes, and this decrease was correlated with the amount of methamphetamine intake. 
Thus, it is possible that newly generated glia in the mPFC during adulthood may play a unique role in functions dependent on the PFC, including learning and goal-directed behavior. Direct evidence for the hypothesis that late or adult-generated glia play an important role in behavior dependent on the PFC will be required. An additional hypothesis that voluntary exercise, a positive factor for mPFC gliogenesis, may reverse the decreases in gliogenesis seen with daily methamphetamine exposure is an exciting future pursuit and warrants detailed investigation. Whether combinations of such opposing factors produce behavioral outcomes is difficult to predict beforehand. Understanding how gliogenesis in the adult brain regions implicated in maintaining functions governing the predisposition to addiction will likely shed light on the basic mechanisms regulating addictionrelated behaviors.

\section{References}

Aguirre AA, Chittajallu R, Belachew S, Gallo V (2004) NG2-expressing cells in the subventricular zone are type C-like cells and contribute to interneuron generation in the postnatal hippocampus. J Cell Biol 165:575-589.

Ahmed SH, Koob GF (1998) Transition from moderate to excessive drug intake: change in hedonic set point. Science 282:298-300.

Alvarez-Buylla A, Lim DA (2004) For the long run: maintaining germinal niches in the adult brain. Neuron 41:683-686.

Alvarez-Buylla A, Herrera DG, Wichterle H (2000) The subventricular zone: source of neuronal precursors for brain repair. Prog Brain Res 127:1-11.

Bacchi CE, Gown AM (1993) Detection of cell proliferation in tissue sections. Braz J Med Biol Res 26:677-687.

Barr AM, Panenka WJ, MacEwan GW, Thornton AE, Lang DJ, Honer WG, Lecomte T (2006) The need for speed: an update on methamphetamine addiction. J Psychiatry Neurosci 31:301-313.

Bartzokis G, Lu PH, Mintz J (2004) Quantifying age-related myelin breakdown with MRI: novel therapeutic targets for preventing cognitive decline and Alzheimer's disease. J Alzheimers Dis 6 [Suppl 6]:S53-S59.

Bayer SA, Altman J (1990) Development of layer I and the subplate in the rat neocortex. Exp Neurol 107:48-62.

Belachew S, Chittajallu R, Aguirre AA, Yuan X, Kirby M, Anderson S, Gallo V (2003) Postnatal NG2 proteoglycan-expressing progenitor cells are intrinsically multipotent and generate functional neurons. J Cell Biol 161:169-186.

Benarroch EE (2005) Neuron-astrocyte interactions: partnership for normal function and disease in the central nervous system. Mayo Clin Proc 80:1326-1338.

Bernier PJ, Bedard A, Vinet J, Levesque M, Parent A (2002) Newly generated neurons in the amygdala and adjoining cortex of adult primates. Proc Natl Acad Sci USA 99:11464-11469.

Cameron HA, McKay RD (2001) Adult neurogenesis produces a large pool of new granule cells in the dentate gyrus. J Comp Neurol 435:406-417.

Corotto FS, Henegar JA, Maruniak JA (1993) Neurogenesis persists in the subependymal layer of the adult mouse brain. Neurosci Lett 149:111-114.

Czeh B, Michaelis T, Watanabe T, Frahm J, de Biurrun G, van Kampen M, Bartolomucci A, Fuchs E (2001) Stress-induced changes in cerebral metabolites, hippocampal volume, and cell proliferation are prevented by antidepressant treatment with tianeptine. Proc Natl Acad Sci USA 98:12796-12801.

Czeh B, Muller-Keuker JI, Rygula R, Abumaria N, Hiemke C, Domenici E, Fuchs E (2007) Chronic social stress inhibits cell proliferation in the adult medial prefrontal cortex: hemispheric asymmetry and reversal by fluoxetine treatment. Neuropsychopharmacology 32:1490-1503.

Dawson MR, Polito A, Levine JM, Reynolds R (2003) NG2-expressing glial progenitor cells: an abundant and widespread population of cycling cells in the adult rat CNS. Mol Cell Neurosci 24:476-488.

Dayer AG, Cleaver KM, Abouantoun T, Cameron HA (2005) New GABAergic interneurons in the adult neocortex and striatum are generated from different precursors. J Cell Biol 168:415-427.

Ehninger D, Kempermann G (2003) Regional effects of wheel running and environmental enrichment on cell genesis and microglia proliferation in the adult murine neocortex. Cereb Cortex 13:845-851.
Goldstein RZ, Volkow ND (2002) Drug addiction and its underlying neurobiological basis: neuroimaging evidence for the involvement of the frontal cortex. Am J Psychiatry 159:1642-1652.

Gould E, Beylin A, Tanapat P, Reeves A, Shors TJ (1999a) Learning enhances adult neurogenesis in the hippocampal formation. Nat Neurosci 2:260-265.

Gould E, Reeves AJ, Graziano MS, Gross CG (1999b) Neurogenesis in the neocortex of adult primates. Science 286:548-552.

Hoehn BD, Palmer TD, Steinberg GK (2005) Neurogenesis in rats after focal cerebral ischemia is enhanced by indomethacin. Stroke 36:2718-2724.

Jentsch JD, Roth RH, Taylor JR (2000) Role for dopamine in the behavioral functions of the prefrontal corticostriatal system: implications for mental disorders and psychotropic drug action. Prog Brain Res 126:433-453.

Kadota T, Kadota K (2004) Neurotoxic morphological changes induced in the medial prefrontal cortex of rats behaviorally sensitized to methamphetamine. Arch Histol Cytol 67:241-251.

Kempermann G (2002) Why new neurons? Possible functions for adult hippocampal neurogenesis. J Neurosci 22:635-638.

Kitamura O, Wee S, Specio SE, Koob GF, Pulvirenti L (2006) Escalation of methamphetamine self-administration in rats: a dose-effect function. Psychopharmacology (Berl) 186:48-53.

Kleim JA, Cooper NR, VandenBerg PM (2002) Exercise induces angiogenesis but does not alter movement representations within rat motor cortex. Brain Res 934:1-6.

Kodama M, Fujioka T, Duman RS (2004) Chronic olanzapine or fluoxetine administration increases cell proliferation in hippocampus and prefrontal cortex of adult rat. Biol Psychiatry 56:570 -580.

Koketsu D, Mikami A, Miyamoto Y, Hisatsune T (2003) Nonrenewal of neurons in the cerebral neocortex of adult macaque monkeys. J Neurosci 23:937-942.

Kondo T, Raff M (2000) Oligodendrocyte precursor cells reprogrammed to become multipotential CNS stem cells. Science 289:1754-1757.

Kornack DR, Rakic P (2001) Cell proliferation without neurogenesis in adult primate neocortex. Science 294:2127-2130.

Lehmann K, Hundsdorfer B, Hartmann T, Teuchert-Noodt G (2004) The acetylcholine fiber density of the neocortex is altered by isolated rearing and early methamphetamine intoxication in rodents. Exp Neurol 189:131-140.

Lin T, Xiang Z, Cui L, Stallcup W, Reeves SA (2006) New mouse oligodendrocyte precursor (mOP) cells for studies on oligodendrocyte maturation and function. J Neurosci Methods 157:187-194.

Madsen TM, Yeh DD, Valentine GW, Duman RS (2005) Electroconvulsive seizure treatment increases cell proliferation in rat frontal cortex. Neuropsychopharmacology 30:27-34.

Magavi SS, Leavitt BR, Macklis JD (2000) Induction of neurogenesis in the neocortex of adult mice. Nature 405:951-955.

Mandyam CD, Norris RD, Eisch AJ (2004) Chronic morphine induces premature mitosis of proliferating cells in the adult mouse subgranular zone. J Neurosci Res 76:783-794.

Markakis EA, Gage FH (1999) Adult-generated neurons in the dentate gyrus send axonal projections to field CA3 and are surrounded by synaptic vesicles. J Comp Neurol 406:449-460.

Mullen RJ, Buck CR, Smith AM (1992) NeuN, a neuronal specific nuclear protein in vertebrates. Development 116:201-211.

Nowakowski RS, Hayes NL (2000) New neurons: extraordinary evidence or extraordinary conclusion? Science 288:771.

Olesen PJ, Nagy Z, Westerberg H, Klingberg T (2003) Combined analysis of DTI and fMRI data reveals a joint maturation of white and grey matter in a fronto-parietal network. Brain Res Cogn Brain Res 18:48-57.

Olson AK, Eadie BD, Ernst C, Christie BR (2006) Environmental enrichment and voluntary exercise massively increase neurogenesis in the adult hippocampus via dissociable pathways. Hippocampus 16:250-260.

Ongur D, Pohlman J, Dow AL, Eisch AJ, Edwin F, Heckers S, Cohen BM, Patel TB, Carlezon Jr WA (2007) Electroconvulsive seizures stimulate glial proliferation and reduce expression of Sprouty 2 within the prefrontal cortex of rats. Biol Psychiatry 62:505-512.

Paulus MP, Hozack NE, Zauscher BE, Frank L, Brown GG, Braff DL, Schuckit MA (2002) Behavioral and functional neuroimaging evidence for prefrontal dysfunction in methamphetamine-dependent subjects. Neuropsychopharmacology 26:53-63.

Paxinos G, Watson C (1997) The rat brain in stereotaxic coordinates. San Diego: Academic. 
Pinilla FG (2006) The impact of diet and exercise on brain plasticity and disease. Nutr Health 18:277-284.

Prudencio C, Abrantes B, Lopes I, Tavares MA (2002) Structural and functional cellular alterations underlying the toxicity of methamphetamine in rat retina and prefrontal cortex. Ann NY Acad Sci 965:522-528.

Ramirez-Amaya V, Marrone DF, Gage FH, Worley PF, Barnes CA (2006) Integration of new neurons into functional neural networks. J Neurosci 26:12237-12241.

Rhodes JS, Ryabinin AE, Crabbe JC (2005) Patterns of brain activation associated with contextual conditioning to methamphetamine in mice. Behav Neurosci 119:759-771.

Sanai N, Tramontin AD, Quinones-Hinojosa A, Barbaro NM, Gupta N, Kunwar S, Lawton MT, McDermott MW, Parsa AT, Manuel-Garcia Verdugo J, Berger MS, Alvarez-Buylla A (2004) Unique astrocyte ribbon in adult human brain contains neural stem cells but lacks chain migration. Nature $427: 740-744$

Sekine Y, Minabe Y, Ouchi Y, Takei N, Iyo M, Nakamura K, Suzuki K, Tsukada H, Okada H, Yoshikawa E, Futatsubashi M, Mori N (2003) Association of dopamine transporter loss in the orbitofrontal and dorsolateral prefrontal cortices with methamphetamine-related psychiatric symptoms. Am J Psychiatry 160:1699-1701.

Sellers DL, Horner PJ (2005) Instructive niches: environmental instructions that confound NG2 proteoglycan expression and the fate-restriction of CNS progenitors. J Anat 207:727-734.
Small GW, Silverman DH, Siddarth P, Ercoli LM, Miller KJ, Lavretsky H, Wright BC, Bookheimer SY, Barrio JR, Phelps ME (2006) Effects of a 14-day healthy longevity lifestyle program on cognition and brain function. Am J Geriatr Psychiatry 14:538-545.

Stewart CV, Plenz D (2006) Inverted-U profile of dopamine-NMDAmediated spontaneous avalanche recurrence in superficial layers of rat prefrontal cortex. J Neurosci 26:8148-8159.

Thompson PM, Hayashi KM, Simon SL, Geaga JA, Hong MS, Sui Y, Lee JY, Toga AW, Ling W, London ED (2004) Structural abnormalities in the brains of human subjects who use methamphetamine. J Neurosci 24:6028-6036.

Van Kampen JM, Hagg T, Robertson HA (2004) Induction of neurogenesis in the adult rat subventricular zone and neostriatum following dopamine $\mathrm{D}_{3}$ receptor stimulation. Eur J Neurosci 19:2377-2387.

van Praag H, Kempermann G, Gage FH (1999) Running increases cell proliferation and neurogenesis in the adult mouse dentate gyrus. Nat Neurosci 2:266-270.

van Praag H, Schinder AF, Christie BR, Toni N, Palmer TD, Gage FH (2002) Functional neurogenesis in the adult hippocampus. Nature 415:1030-1034.

Yu F, Kolanowski AM, Strumpf NE, Eslinger PJ (2006) Improving cognition and function through exercise intervention in Alzheimer's disease. J Nurs Scholarsh 38:358-365. 